\title{
Symmetry constraint of MKdV equations by binary nonlinearization
}

\author{
WEN-XIU MA \\ Institute of Mathematics, Fudan University, \\ Shanghai 2000433, P.R. of China
}

Submitted by A. NIKITIN

Received August 24, 1994

\begin{abstract}
A symmetry constraint for the MKdV integrable hierarchy is presented by binary nonlinearization. The spatial and temporal parts of the Lax pairs and adjoint Lax pairs of $\mathrm{MKdV}$ equations are all constrained as finite-dimensional Liouville integrable Hamiltonian systems, whose integrals of motion are explicitly given. In terms of the proposed symmetry constraint, MKdV equations are decomposed into two finitedimensional Liouville integrable constrained systems and thus a kind of separation of variables for MKdV equations is established.
\end{abstract}

\section{Introduction}

It is well known that there exist close interrelations of symmetries to integrability [1-3]. All classical integrable equations such as KdV, NLS in $(1+1)$-dimensions and KP, DS in $(1+2)$-dimensions were discovered as equations with infinitely many $K$-symmetries $[2$, 4]. Generally speaking, the presence of infinite-dimensional, local $K$-symmetry groups is a characteristic feature of integrable equations in solition theory. Noether's theorem in Lagrangian mechanics and Liouville's theorem in Hamiltonian mechanics may be considered as other important examples of intriguing connections between symmetries and integrability [5]. Liouville's theorem states that an $n$-dimensional Hamiltonian system over some region $\Omega \subseteq R^{2 n}$ with $n$ independent integrals of motion in involution (equivalent to the existence of the $n$-dimensional commutative $K$-symmetry group) is completely integrable, i.e. may be integrated by quadratures.

For soliton theory, investigating the symmetries of equation and symmetry properties of solutions turns out to be fruitful [6-8]. Recently symmetry constraints become prominent because of their important role played. The very successful symmetry constraint

Copyright (C) 1994 by Mathematical Ukraina Publisher. All rights of reproduction in any form reserved. 
method for soliton hierarchies is proposed through the nonlinearization technigue (including mono-nonlinearization [9] and binary nonlinearization [10]). Mono-nonlinearization involves only the Lax pairs for soliton equations. But binary nonlinearization involves both the Lax pairs and adjoint Lax pairs for soliton equations and thus is richer and more systematic. The resulting theory narrows the gap between finite-dimensional Liouville integrable systems and infinite-dimensional integrable soliton equations and provides a method of separation of variables for solving nonlinear soliton equations. Furthermore this kind of symmetry constraints exhibits integrability by quadratures for soliton equations.

In this paper, we shall consider the symmetry constraint of the MKdV integrable hierarchy by binary nonlinearization. The paper is organized as follows. In Section 2, we recall the concrete construction of $\mathrm{MKdV}$ equations beginning with a zero curvature equation and present some basic properties needed in binary nonlinearization. In Section 3 , we perform binary nonlinerization for the Lax pairs and adjoint Lax pairs of the MKdV integrable hierarchy and thus generate a new hierarchy of finite-dimensional, Liouville integrable Hamiltonian systems. Finally in Section 4, we establish a kind of separation of variables for MKdV equations, is to say that we decompose MKdV equations into two integrable, finite-dimensional systems, i.e. one spatial and one temporal systems. This also makes it possible to exactly solve MKdV equations.

\section{MKdV equations and their zero curvature representations}

It is well known that the MKdV integrable hierarchy associates with the following spectral problem

$$
y_{x x}+\left(u_{x}-u^{2}+\alpha\right) y=0 \quad(\alpha \text { is the spectral parameter })
$$

which may be written as

$$
\phi_{x}=U \phi=U(u, \alpha) \phi, \quad U=\left(\begin{array}{cc}
u & \alpha \\
-1 & -u
\end{array}\right), \quad \phi=\left(\begin{array}{l}
\phi_{1} \\
\phi_{2}
\end{array}\right) .
$$

In order to derive the MKdV integrable hierarchy by using the zero curvature equation, we first solve the adjoint representation equation (see ref. [11]) $V_{x}=[U, V]$ of (2.2). Let us choose

$$
V=\left(\begin{array}{cc}
a & b \alpha \\
c & -a
\end{array}\right)
$$

It is easy to see that

$$
[U, V]=\left(\begin{array}{cc}
(b+c) \alpha & 2 u b \alpha-2 a \alpha \\
-2 a-2 u c & -(b+c) \alpha
\end{array}\right)
$$

and thus we find that the adjoint representation equation $V_{x}=[U, V]$ reads

$$
\left\{\begin{aligned}
a_{x} & =(b+c) \alpha \\
b_{x} \alpha & =2 u b \alpha-2 a \alpha \\
c_{x} & =-2 a-2 u c .
\end{aligned}\right.
$$


On setting $a=\sum_{i \geq 0} a_{i} \alpha^{-i}, b=\sum_{i \geq 0} b_{i} \alpha^{-i}, c=\sum_{i \geq 0} c_{i} \alpha^{-i}$, (2.3) engenders equivalently

$$
\left\{\begin{array}{l}
b_{0}+c_{0}=0, \\
a_{i x}=b_{i+1}+c_{i+1}, \quad i \geq 0 \\
b_{i x}=2 u b_{i}-2 a_{i}, \quad i \geq 0 \\
c_{i x}=-2 a_{i}-2 u c_{i}, \quad i \geq 0
\end{array}\right.
$$

We consider three initial equations

$$
b_{0}+c_{0}=0, \quad b_{0 x}=2 u b_{0}-2 a_{0}, \quad c_{0 x}=-2 a_{0}-2 u c_{0} .
$$

The second and third equations yield $\left(b_{0}-c_{0}\right)_{x}=0$. Therefore we have the unique choice $b_{0}=1, c_{0}=-1$ up to a temporal function factor. At the same time, $a_{0}=u$. Throughout this paper, we choose

$$
a_{0}=u, \quad b_{0}=1, \quad c_{0}=-1,
$$

and further we assume that $\left.a_{i}\right|_{u=0}=\left.b_{i}\right|_{u=0}=\left.c_{i}\right|_{u=0}=0, \quad i \geq 1$, which means to select constants of integration to be zero. Because we easily obtain

$$
\begin{aligned}
& b_{i+1, x}-c_{i+1, x}=2 u\left(b_{i+1}+c_{i+1}\right)=2 u a_{i x}, \\
& b_{i+1}-c_{i+1}=2 \partial^{-1} u \partial a_{i}, \quad i \geq 0,
\end{aligned}
$$

the equality (2.4) results in a recursion relation to determine $a_{i}, b_{i}, c_{i}$ :

$$
\left\{\begin{array}{l}
a_{i+1}=L a_{i}, L=-\frac{1}{4} \partial^{2}+u \partial^{-1} u \partial, \quad i \geq 0, \\
b_{i+1}=\frac{1}{2} a_{i x}+\partial^{-1} u \partial a_{i}, \quad i \geq 0, \\
c_{i+1}=\frac{1}{2} a_{i x}-\partial^{-1} u \partial a_{i}, \quad i \geq 0,
\end{array}\right.
$$

Here the first relation is determined by $a_{i+1}=u b_{i+1}-\frac{1}{2} b_{i+1, x}$ and $b_{i+1}=\frac{1}{2} a_{i x}+\partial^{-1} u \partial a_{i}$. By using (2.6), for example, we can work out

$$
\begin{aligned}
& a_{1}=-\frac{1}{4} u_{x x}+\frac{1}{2} u^{3}, \quad b_{1}=-\frac{1}{2} u_{x}+\frac{1}{2} u^{2}, \quad c_{1}=\frac{1}{2} u_{x}-\frac{1}{2} u^{2} ; \\
& a_{2}=\frac{1}{16} u_{x x x x}-\frac{5}{8} u^{2} u_{x x}-\frac{5}{8} u u_{x}^{2}+\frac{3}{8} u^{5}, \\
& b_{2}=-\frac{1}{8} u_{x x x}+\frac{3}{4} u^{2} u_{x}+\frac{1}{4} u u_{x x}+\frac{1}{8} u_{x}^{2}+\frac{3}{8} u^{4}, \\
& c_{2}=-\frac{1}{8} u_{x x x}+\frac{3}{4} u^{2} u_{x}+\frac{1}{4} u u_{x x}-\frac{1}{8} u_{x}^{2}-\frac{3}{8} u^{4} .
\end{aligned}
$$

Let us now associate with the MKdV spectral problem (2.2) the following auxiliary problem

$$
\begin{gathered}
\phi_{t_{n}}=V^{(n)} \phi=V^{(n)}(u, \alpha) \phi, \\
V^{(n)}=\left(\alpha^{n} V\right)_{+}-\left(\begin{array}{cc}
0 & b_{n+1} \\
0 & 0
\end{array}\right)=\left(\begin{array}{cc}
\left(a \alpha^{n}\right)_{+} & \left(b \alpha^{n}\right)_{+} \alpha \\
\left(c \alpha^{n}\right)_{+} & -\left(a \alpha^{n}\right)_{+}
\end{array}\right), \quad n \geq 0 .
\end{gathered}
$$

Where the symbol + denotes the choice of non-negative power of $\alpha$. Then the zero curvature equations

$$
U_{t_{n}}-V_{x}^{(n)}+\left[U, V^{(n)}\right]=0, \quad n \geq 0,
$$


lead to isospectral $\left(\alpha_{t_{n}}=0\right)$ integrable MKdV equations

$$
u_{t_{n}}=K_{n}=a_{n x}=\Phi^{n} u_{x}, \quad n \geq 0,
$$

where the operator $\Phi$ reads

$$
\Phi=L^{*}=-\frac{1}{4} \partial^{2}+\partial u \partial^{-1} u .
$$

It is a hereditary symmetry operator [6] satisfying either of

$$
\begin{aligned}
& \Phi^{2}[K, S]+[\Phi K, \Phi S]-\Phi\{[K, \Phi S]+[\Phi K, S]\}=0, \\
& \Phi^{\prime}[\Phi K] S-\Phi^{\prime}[\Phi S] K-\Phi\left\{\Phi^{\prime}[K] S-\Phi^{\prime}[S] K\right\}=0
\end{aligned}
$$

for arbitrary vector fields $K, S$. The first two nonlinear systems in the MKdV integrable hierarchy $(2.8)$ are as follows

$$
\begin{gathered}
u_{t_{1}}=-\frac{1}{4} u_{x x x}+\frac{3}{5} u^{2} u_{x}, \\
u_{t_{2}}=-\frac{1}{16} u_{5 x}-\frac{5}{2} u u_{x} u_{x x}-\frac{5}{8} u^{2} u_{x x x}+\frac{15}{8} u^{4} u_{x} .
\end{gathered}
$$

Here the first equation is exactly the MKdV equation.

Bi-Hamiltonian structures of MKdV equations in the hierarchy (2.8) may be established by applying a powerful tool, i.e. the so-called trace identity proposed by $\mathrm{Tu}$ [12]. As is usual, we first need the following quantities which are easy to calculate:

$$
<V, \frac{\partial U}{\partial \alpha}>=c, \quad<V, \frac{\partial U}{\partial u}>=2 a,
$$

where $\langle\cdot, \cdot\rangle$ stands for the Killing form of matrices: $\langle A, B\rangle=\operatorname{tr}(A B)$. Then by means of the trace identity [12]

$$
\frac{\delta}{\delta u}<V, \frac{\partial U}{\partial \alpha}>=\alpha^{-\gamma} \frac{\partial}{\partial \alpha} \alpha^{\gamma}<V, \frac{\partial U}{\partial u}>, \quad \gamma=\text { const },
$$

we arrive at

$$
\frac{\delta c}{\delta u}=2 \alpha^{-\gamma} \frac{\partial}{\partial \alpha} \alpha^{\gamma} a .
$$

After comparing the coefficients of $\alpha^{-n}$ on two sides of (2.11), we obtain

$$
\frac{\delta c_{n}}{\delta u}=2(-\gamma-n+1) a_{n-1}, \quad n \geq 1 .
$$

The equality (2.12) with $n=1$ leads to the constant $\gamma=-\frac{1}{2}$. Noticing (2.4) and $\operatorname{Ker} \frac{\delta}{\delta \mathrm{u}}=$ $\operatorname{Im} \partial$, we have

$$
\frac{\delta c_{n}}{\delta u}=\frac{\delta b_{n}}{\delta u}, \quad n \geq 0,
$$

and thus the equality (2.12) gives rise to an important formula

$$
a_{n}=\frac{\delta H_{n}}{\delta u}, \quad H_{n}=\frac{b_{n+1}-c_{n+1}}{2(2 n+1)}, \quad n \geq 0,
$$


which shows that the MKdV hierarchy (2.8) posesses the Hamiltonian structure

$$
u_{t_{n}}=K_{n}=a_{n x}=J G_{n}=J L^{n} a_{0}=J \frac{\delta H_{n}}{\delta u}, J=\partial, \quad n \geq 0 .
$$

We can prove by direct calculation that $J$ and $J L(=\Phi J)$ constitute a Hamiltonian pair and thus the MKdV hierarchy (2.14) is a typical, integrable bi-Hamiltonian hierarchy. But the second Hamiltonian operator

$$
M=J L=-\frac{1}{4} \partial^{3}+\partial u \partial^{-1} u \partial
$$

is non-local. By the above bi-Hamiltonian structure (2.14), we see that the flows determined by (2.8) commute mutually, and each system in the MKdV hierarchy (2.8) has infinitely many symmetries $\left\{K_{n}\right\}_{n=0}^{\infty}$ and conserved quantities $\left\{H_{n}\right\}_{n=0}^{\infty}$.

In order to discuss binary nonlinearization, we need to exhibit the following properties. First, from $\left(V^{2}\right)_{x}=\left[U, V^{2}\right]$, we see that

$$
\left(\frac{1}{2} \operatorname{tr} V^{2}\right)_{x}=\left(a^{2}+b c \alpha\right)_{x}=0 .
$$

Thus we have

$$
a^{2}+b c \alpha=-\alpha,
$$

by observing $\left.\left(a^{2}+b c \alpha\right)\right|_{u=0}=-\alpha$. By the way, we point out that by mathematical induction the local property of $a_{i}, b_{i}, c_{i}$ may be derived from (2.15). This is because besides (2.4) we have

$$
b_{i+1}-c_{i+1}=\sum_{j=0}^{i} a_{j} a_{i-j}+\sum_{j=1}^{i} b_{j} c_{i+1-j}, \quad i \geq 1,
$$

by using (2.15). Second, we can verify

$$
V_{t_{n}}=\left[V^{(n)}, V\right], \quad n \geq 0,
$$

when $u_{t_{n}}=K_{n}$, i.e.

$$
U_{T_{n}}-V_{x}^{(n)}+\left[U, V^{(n)}\right]=0, \quad n \geq 0 .
$$

In fact, we may directly deduce that $V_{t_{n}}-\left[V^{(n)}, V\right]$ satisfies the adjoint representation equation $\phi_{x}=U \phi$ and that

$$
\left.\left(V_{t_{n}}-\left[V^{(n)}, V\right]\right)\right|_{u=0}=0 .
$$

Therefore (2.17) follows from the uniqueness (i.e. if $W_{x}=[U, W]$ and $\left.W\right|_{u=0}=0$, then $W$ itself vanishes) of solutions to the adjoint representation equation. In the next section, the equality (2.17) shall be used to elucidate the commutability of the constrained flows by binary nonlinearization. Bisides, we may similarly get the Zakharov-Shabat equation

$$
V_{t_{m}}^{(n)}-V_{t_{n}}^{(m)}+\left[V^{(n)}, V^{(m)}\right]=\left(V^{(n)}\right)^{\prime}\left[K_{m}\right]-\left(V^{(m)}\right)^{\prime}\left[K_{n}\right]+\left[V^{(n)}, V^{(m)}\right]=0 .
$$

This is to say that $V^{(n)}, n \geq 0$, constitute a commutative Lax operator algebra [13]. 


\section{Binary nonlinearization}

In this section, we want to perform binary nonlinearization [10] for the Lax pairs and adjoint Lax pairs of the MKdV integrable hierarchy (2.8). Note that MKdV equations in the hierarchy (2.8) have another kind of zero curvature representations

$$
\left\{\begin{array}{l}
\psi_{x}=-U^{T} \psi=-U^{T}(u, \alpha) \psi, \\
\psi_{t_{n}}=-\left(V^{(n)}\right)^{T} \psi=-\left(V^{(n)}\right)^{T}(u, \alpha) \psi,
\end{array}\right.
$$

where $T$ means the transpose of a matrix, because $U_{t_{n}}-V^{(n)}+\left[U, V^{(n)}\right]=0$ if and only if $\left(-U^{T}\right)_{t_{n}}-\left(-\left(V^{(n)}\right)^{T}\right) x+\left[-U^{T},-\left(V^{(n)}\right)^{T}\right]=0$. Let us calculate the variational derivative of $\alpha=\alpha(u)$ which respect to the potential beginning with [14]

$$
\left\{\begin{array}{l}
\psi_{x}=U \phi=U(u, \alpha) \phi \\
\psi_{x}=-\left(U^{T} \psi=-U^{T}(u, \alpha) \psi\right.
\end{array}\right.
$$

Making a Gateaux derivative of $\phi_{x}=U(u, \alpha) \phi$ at a direction $K$ leads to the following

$$
\left(\phi^{\prime}[K]\right)_{x}=\left(\frac{\partial U}{\partial u} K+\frac{\partial U}{\partial \alpha} \alpha^{\prime}[K]\right) \phi+U \phi^{\prime}[K] .
$$

By means of $\psi_{x}=-U^{T}(u, \alpha) \psi$, we arrive at

$$
\int_{-\infty}^{\infty} \psi^{T}\left(\frac{\partial U}{\partial u} K+\frac{\partial U}{\partial \alpha} \alpha^{\prime}[K]\right) \phi d x=0 .
$$

Therefore we have

$$
\alpha^{\prime}[K]=\frac{1}{-\int_{-\infty}^{\infty}<\phi \psi^{T}, \frac{\partial U}{\partial \alpha}>d x} \int_{-\infty}^{\infty}<\phi \psi^{T}, \frac{\partial U}{\partial u}>K d x
$$

which implies that the variational derivative of $\alpha$ with respect to the potential $u$ reads

$$
\frac{\delta \alpha}{\delta u}=\frac{<\phi \psi^{T}, \frac{\partial U}{\partial u}>}{-\int_{-\infty}^{\infty}<\phi \psi^{T}, \frac{\partial U}{\partial \alpha}>d x} .
$$

On writing $\psi=\left(\psi_{1}, \psi_{2}\right)^{T}$ and noting (2.2), we acquire

$$
\frac{\delta \alpha}{\delta u}=\frac{1}{E}\left(\phi_{1} \psi_{1}-\phi_{2} \psi_{2}\right), \quad E=-\int_{-\infty}^{\infty} \phi_{2} \psi_{1} d x .
$$

When zero boundary conditions

$$
\lim _{|x| \rightarrow+\infty} \phi=\lim _{|x| \rightarrow+\infty} \psi=0
$$


are imposed, we can verify a simple characteristic property of the variational derivative of $\alpha$ :

$$
L \frac{\delta \alpha}{\delta u}=\alpha \frac{\delta \alpha}{\delta u},
$$

where $L$ and $\delta \alpha / \delta u$ are given by (2.6) and (3.5), respectively.

Let us now discuss two spatial and temporal systems

$$
\begin{aligned}
& \left\{\begin{array}{l}
\left(\begin{array}{l}
\phi_{1 j} \\
\phi_{2 j}
\end{array}\right)_{x}=U\left(u, \alpha_{j}\right)\left(\begin{array}{l}
\phi_{1 j} \\
\phi_{2 j}
\end{array}\right)=\left(\begin{array}{cc}
u & \alpha_{j} \\
-1 & -u
\end{array}\right)\left(\begin{array}{l}
\phi_{1 j} \\
\phi_{2 j}
\end{array}\right), \quad j=1,2, \ldots, N, \\
\left(\begin{array}{l}
\psi_{1 j} \\
\psi_{2 j}
\end{array}\right)_{x}=U\left(u, \alpha_{j}\right)\left(\begin{array}{l}
\psi_{1 j} \\
\psi_{2 j}
\end{array}\right)=\left(\begin{array}{cc}
u & \alpha_{j} \\
-1 & -u
\end{array}\right)\left(\begin{array}{l}
\psi_{1 j} \\
\psi_{2 j}
\end{array}\right), \quad j=1,2, \ldots, N,
\end{array}\right.
\end{aligned}
$$

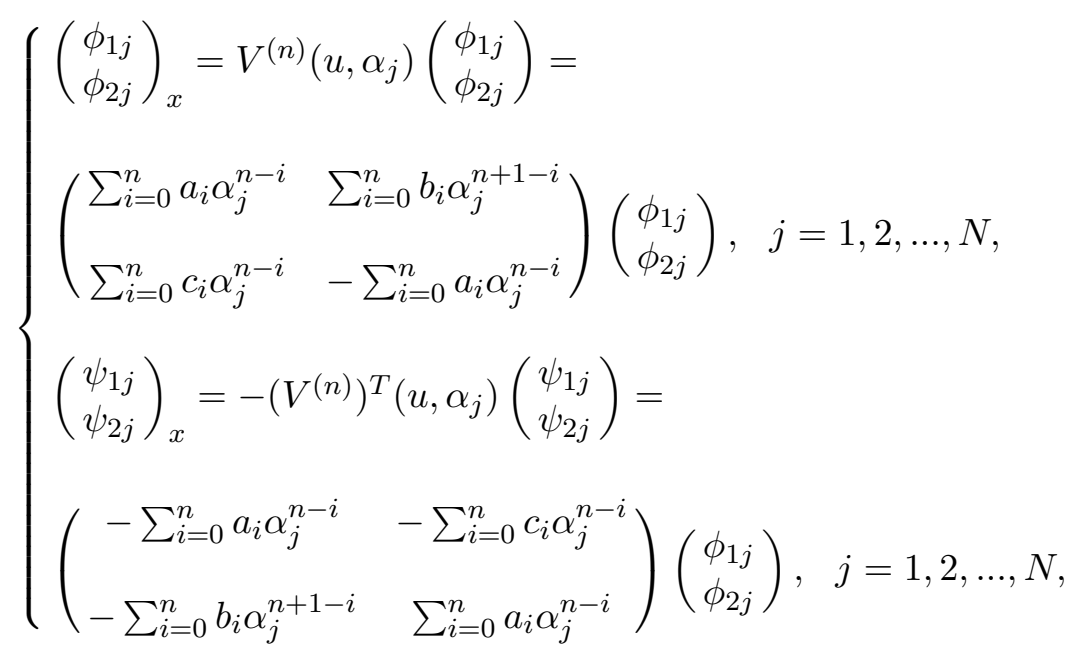

where $\lambda_{1}, \lambda_{2}, \ldots, \lambda_{N}$ and $N$ distinct eigenvalues. The integrability condition of overdetermined linear systems (3.7) and (3.8) is still the $n$-th MKdV equation $u_{t_{n}}=K_{n}$. For (3.7) and (3.8), we may manufacture the following specific symmetry constraints

$$
J G_{m}=J \sum_{j=1}^{N} \frac{1}{2} E_{j} \frac{\delta \lambda_{j}}{\delta u} \quad \text { or } \quad G_{m}=\sum_{j=1}^{N} \frac{1}{2} E_{1} \frac{\delta \lambda_{j}}{\delta u}, \quad E_{1}=-\int_{-\infty}^{\infty} \phi_{2 j} \psi_{1 j} d x, m \geq 0 .
$$

In what follows, we are interested in the Bargmann constraint, which requires the $G$-vector field be a potential linear function not including any potential differential. In our case, the Bargmann constraint reads

$$
J G_{0}=J \sum_{j=1}^{N} \frac{1}{2} E_{j} \frac{\delta \lambda_{j}}{\delta u} \text { or } G_{0}=\sum_{j=1}^{N} \frac{1}{2} E_{1} \frac{\delta \lambda_{j}}{\delta u}
$$

from which the correspondence between the potential and the eigenfunctions and adjoint eigenfunctions particularly clear:

$$
u=f\left(\Phi_{1}, \Phi_{2} ; \Psi_{1}, \Psi_{2}\right)=\frac{1}{2}\left(\left\langle\Phi_{1}, \Phi_{2}\right\rangle-\left\langle\Phi_{1}, \Phi_{2}\right\rangle\right) .
$$


Hereafter for the sake of brevity of presentation, we accept the notation:

$$
\Phi_{i}=\left(\phi_{i 1}, \ldots, \phi_{i N}\right)^{T}, \quad \Psi_{i}=\left(\psi_{i 1}, \ldots, \psi_{i N}\right)^{T}, \quad i=1,2,\langle y, z\rangle=\sum_{j=1}^{N} y_{j} z_{j}
$$

for $y=\left(y_{1}, \ldots, y_{n}\right)^{T}, z=\left(z_{1}, \ldots, z_{n}\right)^{T} \in R^{N}$. The constraint (3.11) is called binary nonlinearization since the function $f$ contains the eigenfunctions and the adjoint eigenfunctions and is nonlinear with respect to them.

Below we will denote by $\tilde{P}$ the expression of $P(u)$ under the binary nonlinear constraint (3.11). The characteristic property (3.6) ensures that

$$
\tilde{a}_{i}=\tilde{L}^{i} \tilde{a}_{0}=\frac{1}{2}\left(\left\langle A^{i} \Phi_{1}, \Psi_{1}\right\rangle-\left\langle A^{i} \Phi_{2}, \Phi_{2}\right\rangle\right), \quad i \geq 0,
$$

and further from (2.6) we can obtain

$$
\begin{gathered}
\tilde{b}_{i}=\left\langle A^{i-1} \Phi_{1}, \Psi_{2}\right\rangle, \quad i \geq 1, \\
\tilde{c}_{i}=\left\langle A^{i} \Phi_{2}, \Psi_{1}\right\rangle, \quad i \geq 1 .
\end{gathered}
$$

At this point, the adjoint representation equation $\tilde{V}_{x}=[\tilde{U}, \tilde{V}]$ remains true. The substitution of (3.11) into the Lax pairs and adjoint Lax pairs (3.7) and (3.8) engenders the nonlinearized Lax pairs and adjoint Lax pairs

$$
\begin{aligned}
& \left\{\begin{array}{l}
\left(\begin{array}{l}
\phi_{2 j} \\
\phi_{1 j}
\end{array}\right)_{x}=U\left(\tilde{u}, \lambda_{j}\right)\left(\begin{array}{c}
\phi_{1 j} \\
\phi_{2 j}
\end{array}\right)=\left(\begin{array}{cc}
\tilde{u} & \lambda_{j} \\
-1-\tilde{u}
\end{array}\right)\left(\begin{array}{c}
\phi_{1 j} \\
\phi_{2 j}
\end{array}\right), j=1,2, \ldots, N, \\
\left(\begin{array}{c}
\psi_{2 j} \\
\psi_{1 j}
\end{array}\right)_{x}=-U^{T}\left(\tilde{u}, \lambda_{j}\right)\left(\begin{array}{c}
\psi_{1 j} \\
\psi_{2 j}
\end{array}\right)=\left(\begin{array}{c}
-\tilde{u}-1 \\
-\lambda_{j} \tilde{u}
\end{array}\right)\left(\begin{array}{c}
\psi_{1 j} \\
\psi_{2 j}
\end{array}\right), j=1,2, \ldots, N ;
\end{array}\right. \\
& \left\{\begin{array}{l}
\left(\begin{array}{l}
\phi_{2 j} \\
\phi_{1 j}
\end{array}\right)_{t_{n}}=V^{(n)}\left(\tilde{u}, \lambda_{j}\right)\left(\begin{array}{c}
\phi_{2 j} \\
\phi_{1 j}
\end{array}\right)= \\
\left(\begin{array}{ll}
\sum_{i=0}^{n} \tilde{a}_{i} \lambda_{j}^{n-i} & \sum_{i=0}^{n} \tilde{b}_{i} \lambda_{j}^{n+i} \\
\sum_{i=0}^{n} \tilde{c}_{i} \lambda_{j}^{n-i} & -\sum_{i=0}^{n} \tilde{a}_{i} \lambda_{j}^{n-i}
\end{array}\right)\left(\begin{array}{l}
\phi_{2 j} \\
\phi_{1 j}
\end{array}\right), j=1,2, \ldots, N, \\
\left(\begin{array}{l}
\psi_{2 j} \\
\psi_{1 j}
\end{array}\right)_{t_{n}}=-\left(V^{(n)}\right)^{T}\left(\tilde{u}, \lambda_{j}\right)\left(\begin{array}{c}
\phi_{2 j} \\
\phi_{1 j}
\end{array}\right)= \\
\left(\begin{array}{cc}
-\sum_{i=0}^{n} \tilde{a}_{i} \lambda_{j}^{n-i} & -\sum_{i=0}^{n} \tilde{c}_{i} \lambda_{j}^{n-i} \\
-\sum_{i=0}^{n} \tilde{b}_{i} \lambda_{j}^{n+1-i} & \sum_{i=0}^{n} \tilde{a}_{i} \lambda_{j}^{n-i}
\end{array}\right)\left(\begin{array}{c}
\phi_{2 j} \\
\phi_{1 j}
\end{array}\right), j=1,2, \ldots, N .
\end{array}\right.
\end{aligned}
$$

Note that the spatial part of the nonlinearized Lax pairs and adjoint Lax pairs, namely the system (3.13) is a system of ordinary differential equations with an independent variable $x$, but the temporal parts of the nonlinearized Lax pairs and adjoint Lax pairs, namely 
the system (3.14) for $n \geq 0$, are all systems of evolution equations with two independent variables $t_{n}, x$. Obviously the system (3.13) may be written out

$$
\left\{\begin{array}{l}
\Phi_{1 x}=\tilde{\Phi}_{1}+A \Phi_{2}=\frac{1}{2}\left(\left\langle\Phi_{1}, \Psi_{1}\right\rangle-\left\langle\Phi_{2}, \Psi_{2}\right\rangle\right) \Phi_{1}+A \Phi_{2}, \\
\Phi_{2 x}=-\tilde{\Phi}_{1}-\tilde{u} \Phi_{2}=-\Phi_{1}-\frac{1}{2}\left(\left\langle\Phi_{1}, \Psi_{1}\right\rangle-\left\langle\Phi_{2}, \Psi_{2}\right\rangle\right) \Phi_{2}, \\
\Phi_{1 x}=-\tilde{u} \Psi_{1}+\Psi_{2}=-\frac{1}{2}\left(\left\langle\Phi_{1}, \Psi_{1}\right\rangle-\left\langle\Phi_{2}, \Psi_{2}\right\rangle\right) \Phi_{1}+\Phi_{2}, \\
\Phi_{2 x}=-A \tilde{\Psi}_{1}+\tilde{u} \Psi_{2}=-A \Psi_{1}+\frac{1}{2}\left(\left\langle\Phi_{1}, \Psi_{1}\right\rangle-\left\langle\Phi_{2}, \Psi_{2}\right\rangle\right) \Psi_{2} .
\end{array}\right.
$$

We want to prove that the system (3.13) is an integrable, finite-dimensional Hamiltonian system in the Liouville sense [5].

Let us now consider the integrability of the spatial part (3.13) and the temporal parts (3.14). The system (3.13) or (3.15) is a cubic nonlinear system and can be represented as the following Hamiltonian form

$$
\Phi_{i x}=\frac{\partial H}{\partial \Psi_{i}}, \Psi_{i x}=-\frac{\partial H}{\partial \Phi_{i}}, \quad i=1,2
$$

with the Hamiltonian function

$$
H=\frac{1}{4}\left(\left\langle\Phi_{1}, \Psi_{1}\right\rangle-\left\langle\Phi_{2}, \Psi_{2}\right\rangle\right)^{2}+\left\langle A \Phi_{2}, \Psi_{1}\right\rangle-\left\langle\Phi_{1}, \Psi_{2}\right\rangle .
$$

We recall that $\tilde{V}_{x}=[\tilde{U}, \tilde{V}]$ holds and thus an obvious equality $\left.\tilde{(} V^{2}\right)_{x}=\left[\tilde{U}, \tilde{V}^{2}\right]$ yields

$$
F_{x}=\left(\frac{1}{2} \operatorname{tr} \tilde{V}^{2}\right)_{x}=\frac{d}{d x}\left(\tilde{a}^{2}+\tilde{b} \tilde{c} \lambda\right)=0
$$

which ensures that $F$ is a generating function of integrals of motion for (3.13) or (3.15). Due to that $F=\sum_{m \geq-1} F_{m} \lambda^{-m}$, we obtain the following formulae for integrals of motion

$$
F_{-1}=\tilde{b}_{0} \tilde{c}_{0}, \quad F_{m}=\sum_{i=0}^{m} \tilde{a}_{i} \tilde{a}_{m-i}+\sum_{i=0}^{m+1} \tilde{b}_{i} \tilde{c}_{m+1-i}, \quad m \geq 0 .
$$

The substitution of (3.12) into the above equality gives out the following explicit expressions for $F_{m}$ :

$$
\begin{gathered}
F_{-1}=-1, F_{0}=\frac{1}{4}\left(\left\langle\Phi_{1}, \Psi_{1}\right\rangle-\left\langle\Phi_{2}, \Psi_{2}\right\rangle\right)^{2}+\left\langle A \Phi_{2}, \Psi_{1}\right\rangle-\left\langle\Phi_{1}, \Psi_{2}\right\rangle=H, \\
F_{m}=\sum_{i=0}^{m} \frac{1}{4}\left(\left\langle A^{i} \Phi_{1}, \Psi_{1}\right\rangle-\left\langle A^{i} \Phi_{2}, \Psi_{2}\right\rangle\right)\left(\left\langle A^{m-i} \Phi_{1}, \Psi_{1}\right\rangle-\right. \\
\left.\left\langle A^{m-i} \Phi_{2}, \Psi_{2}\right\rangle\right)+\sum_{i=1}^{m}\left\langle A^{i-1} \Phi_{1}, \Psi_{2}\right\rangle\left\langle A^{m+1} \Phi_{2}, \Psi_{1}\right\rangle+ \\
\left\langle A^{m+1} \Phi_{2}, \Psi_{1}\right\rangle-\left\langle A^{m} \Phi_{1}, \Psi_{2}\right\rangle, m \geq 1 .
\end{gathered}
$$


At this stage, a direct calculation can lead to

$$
\begin{array}{cl}
\sum_{n \geq 0} \Psi_{i t_{n}} \lambda^{-n}=-\operatorname{tr}\left(\tilde{V} \frac{\partial}{\partial \Phi_{i}} \tilde{V}\right)=-\frac{\partial F}{\partial \Phi_{i}}, \quad i=1,2 \\
\sum_{n \geq 0} \Psi_{i t_{n}} \lambda^{-n}=\operatorname{tr}\left(\tilde{V} \frac{\partial}{\partial \Phi_{i}} \tilde{V}\right)=\frac{\partial F}{\partial \Psi_{i}}, \quad i=1,2
\end{array}
$$

These two equalities enables us to write all the systems (3.14) in the Hamiltonian form

$$
\Psi_{i t_{n}}=-\frac{\partial F_{n}}{\partial \Phi_{i}}, \quad \Phi_{i t_{n}}=\frac{\partial F_{n}}{\partial \Psi_{i}}, \quad i=1,2
$$

where the spatial variable $x$ is treated as a parameter. Note that the simplification of (3.14) to (3.19) is made under the control of (3.13). In order to further show the Liouville integrability of (3.13) and (3.14), we choose the following Poisson bracket

$$
\{P, Q\}=\omega^{2}(I d Q, I d P)
$$

corresponding to the standard symplectic structure on $R^{4 N}$

$$
\omega^{2}=d \Psi_{1} \wedge d \Phi_{1}+d \Psi_{2} \wedge d \Phi_{2}
$$

Here $I d R$ indicates a Hamiltonian vector field of a smooth function $R$ on $R^{4 N}$, defined by $I d R\rfloor \omega^{2}=-d R$, with $\rfloor$ being the left interior product. Because we still have a similar equality

$$
\tilde{V}_{t n}=\left[\tilde{V}^{(n)}, \tilde{V}\right], \quad n \geq 0,
$$

to $(2.17)$, we may see with the same argument that $F=\frac{1}{2} \operatorname{tr} \tilde{V}^{2}$ is also a generating function of integrals of motion for (3.14). Hence $F_{n}, n \geq 0$, are also integrals of motion for (3.14) or (3.19), which means that

$$
\left\{F_{m}, F_{n}\right\}=\frac{\partial}{\partial t_{n}} F_{m}=0, \quad m, n \geq 0 .
$$

This shows that $\left\{F_{n}\right\}_{n=-1}^{\infty}$ constitutes a Poisson algebra, i.e. an involutive system with regard to (3.20), which may also be checked by direct calculation. On the other hand, directly from (3.17) we obtain

$$
\begin{aligned}
& \left.\frac{\partial F_{m}}{\partial \Phi_{1}}\right|_{\Phi_{1}=\Phi_{2}=0}=-A^{m} \Psi_{2},\left.\quad \frac{\partial F_{m}}{\partial \Phi_{2}}\right|_{\Phi_{1}=\Phi_{2}=0}=A^{m+1} \Psi_{2}, \quad m \geq 0, \\
& \left.\frac{\partial F_{m}}{\partial \Psi_{1}}\right|_{\Psi_{1}=\Psi_{2}=0}=A^{m+1} \Phi_{2},\left.\quad \frac{\partial F_{m}}{\partial \Psi_{2}}\right|_{\Psi_{1}=\Psi_{2}=0}=-A^{m} \Phi_{1}, \quad m \geq 0,
\end{aligned}
$$

which implies that there must exist one region $\Omega \subseteq R^{4 N}$ near $\Phi_{1}=\Phi_{2}=0$ or $\Psi_{1}=\Psi_{2}=0$ or near $\Psi_{1}=\Psi_{2}=0$ on which the $2 N$ 1-forms $d F_{i}, \ldots, d F_{2 N+i-1}(i \geq 0)$ are every linearly independent because the Vandermonde determinant $V\left(\lambda_{1}, \lambda_{2}, \ldots, \lambda_{N}\right)$ is non-zero. Therefore according to Liouville's theorem [5], the spatial part (3.13) of the nonlinearized Lax pairs and adjoint Lax pairs, and the temporal parts (3.14) of the nonlinearized Lax 
pairs and adjoint Lax pairs under the control of the spatial part (3.13) are all finitedimensional, integrable Hamiltinian systems, and thus they may be solved by quadratures.

In addition, we remark that under the possible reduction $\Psi_{1}=-\Phi_{2}, \quad \Psi_{2}=\Phi_{1}$, the involutive system $\left\{F_{m}\right\}_{m=-1}^{\infty}$ defined by (3.17) is reduced to

$$
\begin{gathered}
F_{1}=-1, \quad F_{0}=\left\langle\Phi_{1}, \Phi_{2}\right\rangle^{2}-\left\langle A \Phi_{2}, \Phi_{2}\right\rangle-\left\langle\Phi_{1}, \Phi_{1}\right\rangle, \\
F_{m}=\sum_{i=0}^{m}\left\langle A^{i} \Phi_{1}, \Phi_{2}\right\rangle\left\langle A^{m-i} \Phi_{1}, \Phi_{2}\right\rangle-\sum_{i=1}^{m}\left\langle A^{i-1} \Phi_{1}, \Phi_{1}\right\rangle\left\langle A^{m+1-i} \Phi_{2}, \Phi_{2}\right\rangle- \\
\left\langle A^{m+1} \Phi_{2}, \Phi_{2}\right\rangle-\left\langle A^{m} \Phi_{1}, \Phi_{1}\right\rangle, \quad m \geq 1,
\end{gathered}
$$

which forms a Poisson algebra under the symplectic structure $\omega^{2}=d \Phi_{1} \wedge d \Phi_{2}$ on $R^{2 N}$. It corresponds to mono-nonlinearization [15] for the MKdV equations (2.8). All the arguments go parallel for mono-nonlinearization.

\section{Separation of variables}

In the previous section, we have performed the manipulation with binary nonlinearization for the MKdV hierarchy (2.8). It requires zero boundary conditions:

$$
\lim _{|x| \rightarrow+\infty} \Psi_{i}=\lim _{|x| \rightarrow+\infty} \Phi_{i}=0, \quad i=1,2 .
$$

In this section, we should like to tackle the general case where the zero boundary conditions are not imposed. Furthermore we shall present a kind of separation of variables for MKdV equations in the hierarchy (2.8), which also provides a method of solving MKdV equations. In that general case, from (3.2) we can obtain

$$
L \frac{\delta \lambda}{\delta u}=\lambda \frac{\delta \lambda}{\delta u}+I u, \text { namely } L\left(\phi_{1} \psi_{1}-\phi_{2} \psi_{2}\right)=\lambda\left(\phi_{1} \psi_{1}-\phi_{2} \psi_{2}\right)+I u,
$$

where $I$ is an integral of motion of (3.2). Applying (4.1) $m$ times gives rise to

$$
\tilde{a}_{m}=\sum_{i=0}^{m} \frac{1}{2} I_{i}\left(\left\langle A^{m-i} \Phi_{1}, \Psi_{1}\right\rangle-\left\langle A^{m-i} \Phi_{2}, \Psi_{2}\right\rangle\right), \quad I_{0}=1, \quad m \geq 0
$$

where $I_{i}, 1 \leq i \leq m$ are all integrals of motion of (3.13). Further we may work out by (2.6) that

$$
\begin{aligned}
\tilde{b}_{m} & =\sum_{i=0}^{m-1} I_{i}\left\langle A^{m-i-1} \Phi_{1} \Psi_{2}\right\rangle+Q_{m}, \quad m \geq 1, \\
\tilde{c}_{m} & =\sum_{i=0}^{m-1} I_{i}\left\langle A^{m-i} \Phi_{2} \Psi_{1}\right\rangle+R_{m}, \quad m \geq 1,
\end{aligned}
$$

where $Q_{m}$ and $R_{m}$ are all integrals of motion of (3.13), too. The latter two equalities in (2.4) requre the following

$$
Q_{m}=I_{m}, \quad R_{m}=-I_{m}, \quad m \geq 1
$$


This moment, the second equality of (2.4) holds automatically. To determine integrals of motion $I_{i}$, we take advantage of (2.15) or (2.16), namely

$$
\begin{gathered}
\sum_{i=0}^{m} \sum_{k=0}^{i} \frac{1}{2} I_{k}\left(\left\langle A^{i-k} \Phi_{1}, \Psi_{1}\right\rangle-\left\langle A^{i-k} \Phi_{2}, \Psi_{2}\right\rangle\right) \times \sum_{i=0}^{m-i} \frac{1}{2} I_{l}\left(\left\langle A^{m-i-l} \Phi_{1}, \Psi_{1}\right\rangle-\right. \\
\left.\left\langle A^{m-i-l} \Phi_{2}, \Psi_{2}\right\rangle\right)+\sum_{i=1}^{m}\left(\sum_{k=0}^{i-1} I_{k}\left\langle A^{i-k-1} \Phi_{1} \Psi_{2}\right\rangle+I_{i}\right) \times \\
\left(\sum_{l=0}^{m-i} I_{i}\left\langle A^{m+1-i-l} \Phi_{2}, \Psi_{1}\right\rangle-I_{m+1-i}\right)+\left(\sum_{i=0}^{m} I_{i}\left\langle A^{m+1-i} \Phi_{2}, \Psi_{1}\right\rangle-I_{m+1}\right)- \\
\left(\sum_{i=0}^{m} I_{i}\left\langle A^{m-i} \Phi_{1}, \Psi_{2}\right\rangle+I_{m+1}\right)=0, m \geq 1 .
\end{gathered}
$$

On interchanging the summation in the above equality and noting the expressions (3.17) of $F_{m}^{\prime} s$, a direct calculation may yield

$$
I_{m+1}+\frac{1}{2} \sum_{k+l \geq m} I_{k} I_{l} F_{m-(k+l)}-\frac{1}{2} \sum_{i=1}^{m} I_{i} I_{m+1-i}, \quad m \geq 0 .
$$

Evidently $I_{1}=\frac{1}{2} F_{0}$ since $\tilde{a}_{0}^{2}+\tilde{b}_{0} \tilde{c}_{1}+\tilde{b}_{1} \tilde{c}_{0}=0$. Therefore from (4.5), we obtain the following explicit expressions of $I_{m}$ 's:

$$
I_{m}=\sum_{n=1}^{m} d_{n} \sum_{\substack{i_{1}+\cdots+i_{n}=m \\ i_{1}, \ldots, i_{n \geq 1}}} F_{i_{1}-1} \cdots F_{i_{n}-1}, \quad m \geq 1,
$$

where the constants $d_{n}$ are determined recursively by

$$
d_{1}=\frac{1}{2}, \quad d_{2}=\frac{3}{8}, \quad d_{n}=d_{n-1}+\frac{1}{2} \sum_{m=1}^{n-2} d_{m} d_{n-m-1}-\frac{1}{2} \sum_{m=1}^{n-1} d_{m} d_{n-m}, \quad n \geq 3 .
$$

Now we can present the formulae for $\tilde{a}_{m}, \tilde{b}_{m}, \tilde{c}_{m}$ in terms of $F_{i}$, and thus we may directly check that the temporal parts (3.14) of the nonlinearized Lax pairs and adjoint Lax pairs are depicted as the following Hamiltonian systems

$$
\Phi_{i t_{n}}=-\frac{\partial H_{n}}{\partial \Phi_{i}}, \quad \Phi_{i t_{n}}=\frac{\partial H_{n}}{\partial \Psi_{i}}, \quad i=1,2,
$$

where the Hamiltonian functions read

$$
H_{n}=\sum_{m=0}^{n} \frac{d_{m}}{m+1} \sum_{\substack{i_{1}+\cdots+i_{m+1}=n+1 \\ i_{1}, \ldots, i_{m+1} \geq 1}} F_{i_{1}-1} \cdots F_{i_{m+1}-1}\left(d_{0}=1\right), \quad n \geq 0 .
$$

Similarly, we need to point out that the simplification of (3.14) to (4.8) without the zero boundary conditions is made still under the control of the system (3.13). Besides, it is 
easy to find from the commutability of $F_{i}$ that the Hamiltonian phase flows $g_{H_{n}}^{t_{n}}$ defined by the systems (4.8) for $n \geq 0$ commute with each other.

The above manipulation allows us to conclude that the $n$-th equation $u_{t_{n}}=K_{n}$ in the hierarchy (2.8) possesses the following special solution

$$
u\left(x, t_{n}\right)=\frac{1}{2}\left(\left\langle g_{H}^{x} g_{H_{n}}^{t_{n}} \Phi_{1}(0,0), g_{H}^{x} g_{H_{n}}^{t_{n}} \Psi_{1}(0,0)\right\rangle-\left\langle g_{H}^{x} g_{H_{n}}^{t_{n}} \Phi_{2}(0,0), g_{H}^{x} g_{H_{n}}^{t_{n}} \Psi_{2}(0,0)\right\rangle\right),
$$

with $g_{H}^{x}, g_{H_{n}}^{t_{n}}$ being the Hamiltonian phase flows associated with the Hamiltonian systems (3.16) and (4.8), respectively, but $\Phi_{i}(0,0), \Psi(0,0), \quad i=1,2$, being arbitrary constant vectors. It is easy to get that $\left\{H, H_{n}\right\}=0, n \geq 0$. Therefore (4.9) is an involutive solution. In particular, the involutive solution $u\left(x, t_{1}\right)$ given by (4.9) solves the MKdV equation (2.10a). On the other hand, in view of the Liouville integrability of the nonlinearized Lax systems and adjoint Lax systems, this kind of involutive representation of solutions to integrable systems exhibits both the interrelation between $(1+1)$-dimensional integrable systems and finite-dimensional integrable and integrability by quadratures for integrable systems in $(1+1)$-dimensions. Moreover (4.9) provides a kind of separation of variables $x, t_{n}$ for MKdV equations, i.e we can separably solve the Hamiltonian systems (3.16) and (4.8) to find solutions of MKdV equations.

Finally it should be noted that although the above binary nonlinearization method is mathematically systematic, a further investigation should be made for other aspects, for instance, the bi-Hamiltonian structure similar to results by Antonowicz and Wojciechowski et al. [16] of the resulting finite-dimensional systems, appearance of solitons and positons in the involutive solutions, applications of binary nonlinearization to nonisospectral and/or variable coefficient equations (e.g. the NV-MKdV equation [17] and breaking soliton equations [18]), realization of the $\tau$-symmetry constraint by binary nonlinearization, etc.

Acknowledgements: I should like to express my sincerest thanks to Prof. Kai-Seng Chou for inviting me to visit the Chinese University of Hong Kong. I am also grateful to Mr. Kam-Shun Li for valuable discussions. This work was supported by an Earmarked Grant for Research of Hong Kong and by the National Natural Foundation of China.

\section{References}

[1] Fuchssteiner B., Mastersymmetries, Higher Order Time-Dependent Symmetries and Conserved Densities of Nonlinear Evolution Equations, Prog. Theor. Phys., 1983, V.70, 1508-1522.

[2] Fokas A.S., Symmetries and Integrability, Stud. Appl. Math., 1987, V.77, 253-299.

[3] Shabat A.B. and Mikhailov A.V., Symmetries-Test of Integrability, in Important Developments in Soliton Theory (Springer Series in Nonlinear Dynamics), Edited by Fokas A.S. and Zakharov V.E., Springer-Verlag, Berlin, 1993, 355-374.

[4] Ma W.X., $K$-symmetries and $\tau$-symmetries of evolution equations and their Lie algebras, J. Phys. A: Math. Gen., 1990, V.23, 2707-2716;

The algebraic of isospectral Lax operators and applications to integrable equations, Ibid., 1992, V.25, 5329-5343.

[5] Arnold V.I., Mathematical Methods of Classical Mechanics, Springer-Verlag, Berlin, 1978; Dubrovin B.A., Krichever I.M. and Novikov S.P., Integrable systems I, In: Dynamical Systems IV, Ed. by Arnold V.I. and Novikov S.P., Springer-Verlag, Berlin, 1990, 173-280. 
[6] Fuchssteiner B. and Fokas A.S., Symplectic structures, their Bäcklund transformations and hereditary symmetries, Physica D, 1981, V.4, 47-66;

Sokolov V.V., On symmetries of evolution equations, Uspekhi Mat. Nauk, 1988, V.43, 133-163;

Mikhailov A.V., Shabat A.B. and Sokolov V.V., The symmetry approach to classification of integrable equations, in what is Integrability? (Springer Series in Nonlinear Dynamics), Ed. by Zakharov V.E., Springer-Verlag, Berlin, 1990, 115-184.

[7] Ma W.X., An approach for constructing nonisospectral hierarchies of evolution equations, J. Phys. A: Math. Gen., 1992, V.25, L719-L726;

A simple scheme for generating nonisospectral flows from zero curvature representation, Phys. Lett. A, 1993, V.179, 179-185;

Ruan H.Y. and Lou S.Y., New symmetries of the Jaulent-Miodek hierarchy, J. Phys. Soc. Jpn., 1993, V.62, 1917-1921.

[8] Chou K.S. and Wan Tom Y.H., Asymptotic radial symmetry for solutions of $\Delta u+e^{u}=0$ in a punctured disc, to appear in Pacific J. Math., 1994.

[9] Cao C.W., Nonlinearization of the Lax system for AKNS Hierarchy, Sci. China A, 1990, V.33, 528536 ;

Zeng Y.B. and Li Y.S., The constraints of potentials and the finite-dimensional integrable systems, J. Math. Phys., 1989, V.30, 1679-1689;

Cao C.W. and Geng X.G., A nonconfocal generator of involutive systems and three associated soliton hierarchies, J. Math. Phys., 1991, V.32, 2323-2328.

[10] Ma W.X. and Strampp W., A explicit symmetry constraint for the Lax pairs and the adjoint Lax pairs of AKNS systems, Phys. Lett. A, 1994, V.185, 277-286;

Ma W.X., Binary nonlinearization for the Dirac systems, IDMF preprint (9343), 1993.

[11] Fordy A.P. and Gibbons J., Factorization of operators I, II, J. Math. Phys., 1980, V.21, 2508-2510; Ibid, 1981, V.22, 1170-1175.

[12] Tu G.Z., Constrained formal variational calculus and its applications to soliton equations, Scientia sinica A, 1986, V.24, 138-148;

The trace identity, a powerful tool for constructing the Hamiltonian structure of integrable systems, J. Math. Phys., 1989, V.30, 330-338.

[13] Ma W.X., Lax representations and Lax operator algebras of isospectral and nonisospectral hierarchies of evolution equations, J. Math. Phys., 1992, V.33, 2464-2476;

The algebraic structure of zero curvature representations and application to coupled KdV systems, $J$. Phys. A: Math. Gen., 1993, V.26, 2573-2582;

[14] Fokas A.S. and Anderson R.L., On the use of isospectral eigenvalue problems for obtaining hereditary symmetries for Hamiltonian systems, J. Math. Phys., 1982, V.23, 1066-1073.

[15] Gu Z.G., Two finite-dimensional completely integrable Hamiltonian systems associated with the solutions of the MKdV hierarchy, J. Math. Phys., 1991, V.32, 1531-1536.

[16] Antonowicz M. and Wojciechowski S., How to construct finite-dimensional bi-Hamiltonian systems from soliton equations: Jacobi integrable potentials, J. Math. Phys., 1992, V.33, 2115-2125; Blaszak M., Bi-Hamiltonian field Garnier system, Phys. Lett. A, 1993, V.174, 85-88.

[17] Chan W.L. and Li K.S., Non-propagating solitons of the non-isospectral and variable coefficient modified KdV equation, to appear in: J. Phys. A: Math. Gen., 1994, V.27.

[18] Bogoyavlenskii O.I., Breaking solitons in 2+1-dimensional integrable equations, Uspekhi Math. Nauk, 1990, V.45, 17-77;

Breaking solitons V: systems of hydrodynamic type, Math. USSR Izv., 1992, V.38, 439-454. 